\title{
Influence of electric field on the remediation of polluted soil using a biobarrier assisted electro-bioremediation process
}

\author{
E. Mena, J. Villaseñor, P. Cañizares, M.A. Rodrigo* \\ Chemical Engineering Department. Faculty of Chemical Sciences and Technologies \& Research \\ Institute for Chemical and Environmental Technology. Universidad de Castilla La Mancha, \\ Campus Universitario s/n.13071, Ciudad Real, Spain
}

\begin{abstract}
In this work, it was carried out the study of the electro-bioremediation of soil polluted with diesel by means of combined electrokinetic soil flushing and biobarriers, using bench scale setups and fourteen days-long tests. Two different biobarriers were evaluated: one of them was developed in the laboratory, with a culture of diesel-degrading microorganisms supported on gravel particles (BB1); the other was obtained by mixing directly clay soil with activated sludge obtained in a municipal wastewater treatment plant (BB2). Biobarriers were placed in a central point of the soil section to be treated, to prevent the negative consequences of $\mathrm{pH}$ fronts on microorganisms viability. Potential difference of 0.5 and $1.0 \mathrm{~V} \mathrm{~cm}^{-1}$ were applied. For the promotion in the transport of the diesel to the biobarrier, a surfactant solution was used as electrolyte (flushing fluid). Results confirm that negative influence of the extreme $\mathrm{pH}$ fronts on the microbial viability can be prevented using this electro-bioremediation approach. In the same way, uniform diesel removal was obtained all over the soil by using the surfactant solution. After fourteen days of treatment, in the experiments using the BB1, 19.36\% and $27.36 \%$ of the total amount of diesel present in the soil were removed applying 0.5 and $1.0 \mathrm{~V} \mathrm{~cm}^{-1}$, respectively. In the experiments using BB2, $23.33 \%$ and $29.10 \%$ referred to the total amount of diesel were removed after the fourteen days tests at 0.5 and $1.0 \mathrm{~V} \mathrm{~cm}^{-1}$, respectively, indicating that the non-specific barrier is slightly more efficient despite not containing an acclimated culture.
\end{abstract}

\section{Keywords}

Electro-bioremediation, biobarriers, diesel polluted soil, enhanced processes, electrokinetic

\section{Highlights}

- Combined EKSF-biobarrier treatment is successful for the removal of diesel from clay soil. 
- A non-specific biobarrier can be implemented succesfully instead of the autochthonous microorganisms.

- Optimal biological conditions were achieved placing the biobarrier in the central section of soil.

- Higher electric fields applied in electro-bioremediation results in a faster pollutant removal.

- The use of a surfactant solution produces a uniform removal of diesel all over the soil treated.

* Author to whom all correspondence should be addressed: manuel.rodrigo@uclm.es 


\section{Introduction}

According to the recent literature, coupling electrokinetic and bioremediation processes may result in a very effective technology due to the very different nature and targets of both technologies [1-12]. However, there are many different ways to combine the single treatments and each of them results in a very complex process with different efficiencies in the removal of pollutants. For the synergistic "blend" of both treatments, the significant modifications that the application of the electric field may cause in the conditions of the soil must be exhaustively controlled, in order to attain the optimal performance of the biological process. Unfortunately, this is not an easy task and keeping temperature and $\mathrm{pH}$ in suitable values for microbial life is very often a challenge. A very interesting alternative for the combination of electrokinetic soil flushing (EKSF) and bioremediation is based on the concentration of the pollutant-degrading microorganisms population in a limited section, so-called biological permeable reactive barrier or biobarrier, through which the flushing fluid is passed (once or several times) in order to be treated. $[13,14]$

The concept of Permeable Reactive Barrier (PRB) is not new and it was introduced much time ago for soil remediation processes. A PRB is defined as an engineered zone of a reactive material that is placed in the direction of groundwater flow to help intercept a pollution plume that is carried within an aquifer by retaining or degrading the pollutants [15]. The subsurface pollution plume can flow throughout the PRB using the natural hydraulic gradient or a pump-and-treat method. However, when the soil to be treated has very low permeability, as it is the case of the clay soils, the mobilization of the water using these conventional techniques is not possible. In this particular case, electrokinetic (EK) technology could become an interesting alternative in order to transport the pollutants to the barrier, where they are going to be treated. The key component of the barrier technology is the material used for its manufacturing, because it fixes the type of treatment that it is going to be applied. Different materials have been proposed to build PRBs, typically looking for the promotion of several removal mechanisms related to the nature of the pollutants. Most important applications include reduction of halogenated pollutants using elemental metals, adsorption with porous high surface materials, ion exchange with resin-based materials, biological degradation using biological supports, etc.

In this context, numerous works related to the use of PRBs have been published in the last two decades $[14,16-20]$ focused especially on the removal of heavy metals or organic compounds from polluted soils. However, the combination of the EK and the PRB treatments is much more recent and, consequently, nowadays only a limited number of publications are available regarding this topic [21-27]. These publications suggest that it is not only a feasible technology but probably the most promising application of EK technology, because it helps to expand the applicability of the technology to a very wide range of pollutants. 
In this work, the attention is focused on a particular type of barriers based on the promotion of the biological degradation technology [28-34]. These are named Biological Permeable Reactive Barriers or Biobarriers (BB). A biobarrier can be defined as a fixed culture bioreactor that includes a porous supporting material and a microbial biofilm attached on its surface. The working principle of a biobarrier is the same as that of a conventional biofilm reactor. In fact, the only relevant difference to highlight is that the biobarrier is inserted into the soil during EK treatment and that especial attention has to be paid in order to prevent the negative consequences that EK processes can have on the biological culture, in particular the extreme changes in the $\mathrm{pH}$ and temperature.

In this context, the main objective of this work is the evaluation of the combined EKSF with the biological process carried out by the microorganisms contained in a biobarrier. It was evaluated the efficiency obtained using specific and non-specific diesel-degrading biobarriers and, more importantly, the influence of the electric field on the performance of the treatment technology and the optimization of the value of this parameter.

\section{Experimental}

The experimental setup used in these experiments was based on that used in the experiments previously discussed elsewhere [35, 36], and it is schematized in Figure 1. The setup was made of transparent methacrylate and divided into seven compartments. The central compartment was loaded with the corresponding biobarrier for each experiment. On both sides of the biobarrier, the diesel-polluted kaolinite was loaded and compacted manually. Graphite plates, used as anodic and cathodic electrodes, were placed on one side of each section of polluted soil.

Two different biobarriers were evaluated. The development and more important properties of each of them have been previously described in the literature $[35,36]$. One of the biobarriers (socalled biobarrier 1 (BB1)) was composed of gravel particles with the diesel-degrading microbial consortia supported on its surface. The second biobarrier used in the experiments (so-called biobarrier 2 (BB2)) was manufactured by mixing non-polluted kaolinite soil with raw active sludge taken directly from the biological reactors of the municipal Wastewater Treatment Plant of Ciudad Real (Spain). The ratio active sludge/clean soil was $40 \mathrm{wt} \%$.

Taking into account that the biological active areas were placed in an intermediate section of the installation, far away from the electrodes, it was necessary the use of a surfactant in order to promote the transport of the diesel pollutant to the biobarrier. A flushing fluid consisting of a solution of sodium dodecyl sulfate (SDS) surfactant $\left(2.38 \mathrm{~g} \mathrm{dm}^{-3}\right)$ was added in the cathodic compartment. Taking into account that the SDS is an anionic surfactant, the superficial charge of the diesel-SDS micelles is expected to be negative and, consequently, they are expected to be 
transported from the cathodic area towards the anodic compartment, being the diesel oil degraded when passing throughout the biobarrier area.

The duration of the experiments was 2 weeks, long enough to have a clear view of the main processes happening in the soil and short enough to avoid depletion of pollutants (hence allowing quantifying and comparing for different technologies the rate of the remediation). In order to evaluate the influence of the electric field, experiments were carried out at 0.5 and $1.0 \mathrm{~V} \mathrm{~cm}^{-1}$. The sampling procedure and analytical techniques were the same that in previous remediation experiments that have been previously published in the literature $[35,36]$. The parameters monitored were $\mathrm{pH}$, conductivity, moisture content, the concentration of microorganisms and the total-P, total-N, diesel and SDS concentrations. However, in the experiments with the BB1 (diesel-degrading microorganisms), several parameters were also monitored in the nutrient medium of the biobarrier area during the treatment. These were the concentration of inorganic nutrients and dissolved oxygen, $\mathrm{pH}$, conductivity and temperature. The BB2 (activated sludge) was sampled in the same way that the polluted soil treated.

\section{Results and discussion}

In this work, results obtained in the treatment of diesel polluted soil with the combined electrokinetic and permeable reactive biobarriers (so-called BioBarrier Assisted ElectroBioremediation process) are discussed by studying the changes in the more relevant parameters during the 14 days-long tests carried out and explaining the way in which they affect the performance of the remediation process. Next, the main observations are discussed in order to describe the technology

\subsection{Current density}

Electro-bioremediation tests were carried out at constant electric field by setting a potential difference between the anode and cathode placed in the experimental soil sections and keeping it constant during the whole tests. This operation mode (potentiostatic) implies that changes in the soil conductivity are going to be reflected on the resulting current intensity, which, at the same time, will fix the rate of the electrochemical and transport processes. Figure 2 shows the changes in the current density (defined as the relationship between the resulting intensity and the cross section area of the soil to be treated) in the four tests (experiments with the two different biobarriers and at two different electric field values) studied in this work.

As it can be observed, current density values decreases in the four tests during the treatment. These changes are a consequence of the changes produced in the characteristic of the soil and they can be explained in terms of an increase in the electrical resistance of the soil matrix, which can 
be associated with a decrease in the conductivity of the liquid contained. Initially, this decrease is more pronounced in the experiments carried out at the highest value of electric field, and this fact is easy to be explained in terms of the application of a higher electrical charge to the soil. However, as the time goes by, differences are minimized and comparable values are obtained in the four tests after 14 days of operation. There is a variety of reactions that takes part in the soil when an electric field is applied, including oxidation and reduction of water on the electrodes, ion exchange, precipitation / re-dissolution, etc. Most of them directly affect to the ionic species contained in the soil and the decrease in the intensity has to be explained by a decrease in the conductivity, which can be further associated to a significant removal of ions or to the obstruction of their movement by fixation processes. [27, 37-39]

\subsection{Electro-osmotic flow}

The electro-osmotic flow (EOF) is related to the volume of water electrokinetically transported from the anode to the cathode, and it is plotted in Figure 3.

As expected according to the Helmholtz-Smoluchowski equation (Equation 1), the higher the electric field applied $\left(E_{x}\right)$ the higher is the rate of the electro-osmotic flux (EOF), because the dielectric constant $(D)$, vacuum permittivity $\left(\varepsilon_{0}\right)$, fluid viscosity $(\eta)$ are expected to be almost the same in the four tests.

$$
{ }_{\text {E.O.F. }}=-\frac{D \varepsilon_{0} \zeta}{\eta} E_{X}
$$

The changes in the fluxes of water transported by the electro-osmosis process are comparable to those observed in the current density and it indicates that current becomes the driving force of this type of flux. To explain this observation, it has to be accounted that current density affects to the ionic concentration and hence to the Z-potential $(\zeta)$ of the soil, thus explaining the change in the fluxes in agreement with the Helmholtz-Smoluchowski model [39]. In comparing the two types of biobarriers, it can be observed that the EOF is higher in the biobarrier with gravel particles as compared to the biobarries with clay particles. Initially this is not the expected behavior because continuity of the soil is not kept with this type of biobarrier and because differences observed in the resulting current densities were not as clear.

\subsection{Changes in the operating conditions within the biobarriers}


In the experiments carried out, the pollutant-degrading microbial population is concentrated in a specific section of the soil to be treated where the biobarrier is set up. In this way, it is crucial for the successful treatment of the polluted soil to assure that conditions in the biobarrier section have optimal values for the microbial activity. Figure 4 focuses on the variation of different parameters monitored in the biobarrier section. In this point, it is important to point out the singular characteristics of each biobarrier. The biobarrier with the diesel-degrading microorganisms (BB1) was a porous gravel bed continuously submerged in nutrient medium, so it was easy to take liquid samples from the pore water without modifying significantly process conditions. However, by using the biobarrier made with the clean soil mixed with the activated sludge (BB2), it was not possible to carry out the same sampling procedure and rhizoms were needed to take liquid samples modifying importantly the flow conditions of the test. For this reason, in this second case, solid samples of the biobarrier were only obtained at the beginning and at the end of the treatment.

The first parameter with great influence in the biodegradation process in soils is the temperature which should be kept during the treatment within a narrow range because extreme temperature affects seriously to the microorganisms activity. Figure 4.a, shows the changes observed in the temperature of the biobarrier area during the four tests. As it can be observed, temperature in the biobarriers was maintained around the room value during the four remediation tests. This fact can be explained in terms of the low current density values monitored.

Another parameter monitored in the biobarrier zone, with great influence on the operation of the biological process is the $\mathrm{pH}$. Changes during the tests are shown in Figure 4.b (only for BB1). As it can be observed, $\mathrm{pH}$ value in the biobarrier was maintained around neutral point during the whole tests. This observation confirms that the location of the biobarrier in the central section of the soil was a good strategy to minimize the influence of the $\mathrm{pH}$ fronts on the biobarrier medium and to allow microorganisms to keep suitable conditions for life not only from the viewpoint of temperature but also from the point of view of $\mathrm{pH}$.

A different parameter of great relevance in the combined electrokinetic and biobarriers treatment is the availability of dissolved oxygen for the aerobic degradation of the pollutant. On the surface of the anodic electrode, due to the water oxidation reaction, great amounts of oxygen are electrochemically generated. However, due to the low permeability value of the soil, the diffusion of oxygen throughout the soil was not favored [41]. In Figure 4.c (only for BB1), it is plotted the variation in the dissolved oxygen concentration in the liquid medium with the diesel-degrading microorganisms supported on gravel. As it can be observed, dissolved oxygen concentration remained around the same value during the complete tests, very close to the saturation concentration with only slight fluctuations. This result indicated that the concentration of oxygen in the biobarrier medium was high enough to assure the aerobic degradation of the diesel oil pollutant. It is important to note that the previous development of the microorganisms attached in 
the biobarrier was conducted under flooded and non-aerated conditions. Thus, in its application for soil treatment, high dissolved oxygen concentrations would not be necessary for the diesel biodegradation process. However, aerobic processes are usually more efficient, which could be relevant for the application of this technology in similar aerobic remediation processes.

Finally, it is worth paying attention on the conductivity variation in the liquid nutrient medium of the biobarrier, represented in Figure 4.d for BB1. It is observed a clear decrease in the ionic conductivity of the liquid contained in the biobarrier during the two tests, with values much lower than those added with the Bushnell Hass Broth (BHB) nutrient media. At this point, it is worth reminding that this nutrient medium was not only added at the beginning of the treatment but also during the remediation tests because level of liquid in the biobarrier was maintained constant with the addition of the volume required of BHB solution. Hence, the decrease in the ionic conductivity implies a removal of the nutrients added and this removal has to be explained in terms of three different mechanisms: a) consumption of ionic species by microorganisms, b) transport by electrokinetic processes (including drag with the electro-osmotic flow and electromigration) and c) removal with samples taken during monitoring.

\subsection{Changes in the electrolytes}

Figure 5 shows the changes in the $\mathrm{pH}$ of the anolyte and catholyte during the electrobioremediation processes with biobarriers.

In these experiments, it was not added a buffer solution in the electrolyte because the position of the concentrated pollutant-degrading barrier was selected trying to avoid the negative influence of the extreme $\mathrm{pH}$ fronts. As it is observed, $\mathrm{pH}$ underwent variation to acid value in the anolyte and to basic value in the catholyte from the beginning of the tests. However, it is expected that the influence of these extreme values in the viability of the microbial population would not be very important.

Figure 6 shows the changes in the conductivity of the anolyte and catholyte in the electrobioremediation tests with biobarriers. A general increase in both the anolyte and catholyte was observed, mainly due to the electromigration processes of the ions towards the anodic and cathodic wells. In these experiments, it is expected that the electromigration of the inorganic nutrients added in the biobarrier area to favor the biological degradation was the predominant cause of the increase in the conductivity of the electrolytes.

\subsection{Soil after the remediation tests}

After the fourteen days long tests a postmortem analysis was carried out in order to map (3-D) the more relevant parameters in the soil including moisture, conductivity, $\mathrm{pH}$, microbial concentration 
and diesel concentration. This analysis is expected to become a clear indicative of the achievements which may be carried out with this technology.

The study of the variation in the moisture conditions of the soil during the electro-bioremediation tests is very important. The successful application of the electrokinetic technology is only possible with high values of moisture in the soil treated. Likewise, the biodegradation process requires moisture because it primarily happens in the aqueous phase of the system. During the application of the electrokinetic treatment, there are two important processes that modify the water content of the system: the electro-osmotic and the evaporation processes. Figure 7 shows the variation in the moisture values of the soil with the treatment for the two types of biobarrier studied in this work. At the beginning of the treatment tests, the moisture of the soil was near to the saturation point (around 40\%). After the 14-days treatment, the moisture was kept close to the initial value or it shows a slight decrease. The most important changes were obtained in the tests carried out under the highest electric field. In these cases, also higher volumes of water were transported by the electro-osmosis processes according to Figure 3. This fact points out the importance of the EOF in the moisture content of the soil. On the other hand, taking into account that temperature is kept under the same range in the four tests, evaporation process is expected to affect to the moisture content of the soil in the same extension, so it can be indicated that the most important phenomena affecting the moisture of the soil is the electro-osmosis. In comparing the changes observed for the two biobarriers, it can be stated that changes seem to be greater for BB2 suggesting that more relevant processes are happening in this type of biobarrier regarding water transport. In this point, it is important to remember that texture of the two biobarriers is completely different and continuity is only kept for BB2, because of the huge pore size of the gravel as compared to that of the clay.

Figure 8 shows the initial and final values of the conductivity measured in different sections of the soil (including biobarriers) when the four electro-bioremediation tests are applied.

As it was expected, a general decrease in the conductivity of the soil was observed, which matches with the decrease in the current density observed during the tests and can be related to the decrease in the ionic species concentration caused by electrokinetic processes (concentration in the wells and extraction with the flushing fluid). The decrease is more important under the lowest electric field applied and when high electric fields are applied it seems more a redistribution of conductivity rather than a real removal.

Successful combination of the electrokinetic processes and the biological permeable reactive barriers directly depends on the possibility to maintain suitable conditions in the soil for the microorganisms, which allows them to carry out the degradation of the diesel oil pollutant. The $\mathrm{pH}$ is one of the variables more importantly affected in soils that undergo an electrokinetic treatment. Figure 9 shows the variations in the value of $\mathrm{pH}$ of the soil before and after the remediation treatment tests. 
A clear influence of the acidic and alkaline fronts moving throughout the soil matrix was observed in the $\mathrm{pH}$ profiles. A marked decrease in the $\mathrm{pH}$ values in the sections near to the anode was obtained and also, less significant increase is observed near the cathode. Due to the faster transport of the acid front, a much more extended portion of the soil was affected by the decrease in the $\mathrm{pH}$. Anyway, $\mathrm{pH}$ in the biobarrier section was perfectly controlled with no actions (except for the initial choice in the position) for both types of biobarriers, and this help to obtain in this zone the best performance conditions for the microorganisms responsible for the degradation of the pollutant.

By using the combined electrokinetic and biobarrier technology, the existence of suitable conditions for the microbial survival in soil areas different to the biobarrier is not absolutely necessary. However, the evaluation of the population of microorganisms in different sections of the soil has great interest in order to check the harshness of the treatment technology and Figure 10 shows the variation in the population of microorganisms alive in the soil after the treatment. After the treatment, marked microbial concentration profiles were obtained. Regarding the biomass growth, at the end of the experiment, higher microbial concentrations were observed in the central areas near the biobarriers in which the diesel-degrading microorganisms were supported. Furthermore, the total volatile solids concentration in the BB1 was measured. This concentration was directly related to the biomass concentration that was supported in the biobarrier. Overall, the amount of biomass attached to the BB1 increased by $25 \%$ after the tests. In this area (as previously discussed), conditions are optimal for microbial growth in terms of the $\mathrm{pH}$ and nutrient concentrations. This result indicates the presence of biological activity in the area of the biobarrier. On the other hand, it was observed displacement of microorganisms to the sections placed near to the electrodic wells, although lower microbial concentrations were obtained. The presence of microorganisms in zones that were far from the central biobarrier could be caused by the biofilm detachment process (BB1) and the EK transport of the microorganisms (in both cases). The biofilm detachment process resulted from biomass growth and the movement of water through the biobarrier. This fact would explain the occurrence of microorganisms in the sections surrounding the biobarriers. Finally, the extreme $\mathrm{pH}$ reached near to the electrodes prevents microbial performance and explains the much lower concentration of microorganisms, near to the electrode wells.

There is an important difference in comparing electro-bioremediation technologies with the pollutant-degrading microorganisms supported on a biobarrier or dispersed all over the soil treated and it is directly related to the possibilities of interaction among the different species taking part in the degradation process (microorganisms, nutrients, electron acceptors and pollutants). In case of biobarriers, it is necessary the displacement of the pollutant toward the biodegradation sections and, for non-charged pollutants, this implies the assistance of reagents for improving the transport of the pollutant such as surfactants. Based on this fact, in these 
experiments, an anionic surfactant solution containing SDS was used as flushing fluid and it was added to the catholyte at the beginning of the experiments. It was chosen based on the previous experience of the research group about the transport of organic pollutants which demonstrated that SDS is a very good choice because it is not irreversibly fixed in the soil [42, 43]. In addition, it is biodegradable and behaves as a good extraction agents in both flushing and washing processes. The addition of this surfactant makes possible the transport of micelles containing the diesel oil molecules toward the biobarrier section by the electrophoresis and by the dragging with the electro-osmosis flow. Figure 11 shows the surfactant concentration profile in the soil at the end of the 14 days long electro-bioremediation treatment. At the beginning of the treatment there was not surfactant distributed in the soil, because it was added only in the cathode well. This fact means that initial concentration through the soil was zero in all positions and that concentration measured in the post mortem analysis has to be explained in terms of transport of the surfactant molecule during the tests. As it can be observed, after the 14 days treatment tests a distribution of surfactant was obtained in the four soil sections, with a clear dependence on the distance to the cathode.

The soil sections placed near to the cathodic compartment exhibited the highest concentrations of surfactant. As the distance to the cathodic compartment increases, a much lower surfactant concentration is detected. Anyway, in all the soil sections, the presence of the surfactant was detected. This fact clearly indicates that surfactant is transported efficiently by electromigration and that, if it combines with diesel, then micelles can be formed and contribute to the transport of diesel from the soil to the electrode wells, by dragging of the electro-osmotic flux or by electrophoresis. These different transport processes affecting the distribution of surfactant in the soil may have as ultimate consequence the practically uniform removal of diesel oil pollutant all over the soil.

In Figure 12, the changes in the diesel concentration profiles in the soil before and after the treatment tests are represented. This is the most important parameter evaluated in the experiments, because it allows us the quantification of the efficiency of the treatment technology. As it can be observed, an important removal of diesel is obtained in the four tests. Results indicate that a homogeneous removal of pollutants was achieved in the soil (diesel removal efficiencies were $19.36 \%, 27.36 \%, 23.33 \%, 29.10 \%$ in cases a, b, c and d respectively). Because the higher volatile hydrocarbon fractions were previously removed and the increase in soil temperature was negligible, hydrocarbon volatilization was not considered, and only the combination of biological and EK phenomena was assumed to be responsible for the diesel removal. Moreover, no diesel oil was detected in the electrodic wells. The homogeneity of the diesel removal would result from two aspects. First, the microorganisms were present nearly throughout the soil portion (not only the biobarrier) due to the biofilm detachment and the EK transport. In addition, the diesel was emulsified with SDS and moved across the soil because of a combination of electrokinetic 
phenomena (as discussed before) and it would allow for the mobilization of the diesel through the biobarrier, where higher degradation activity would be conducted. The combination of electromigration towards the anode and electro-osmosis towards the cathode would produce the movement of SDS/diesel micelles across the whole soil, passing through the biobarrier in both directions.

In comparing a previous study regarding diesel biodegradation with the same microbial consortia [44], the biodegradable fraction of the chemical oxygen demand (COD) of the diesel that was used was approximately $73 \%$. This result indicates that an important amount of the biodegradable fraction of the diesel hydrocarbons $(28.32 \%, 37.48 \%, 34.54 \%$ and $39.86 \%$ in cases a, b, c and d respectively) were removed trough the EK/biobarrier treatment in two weeks. It has been also observed that the higher the electric field, the higher is the resulting removal of diesel with both types of biobarriers. In the case of BB1, a great and random axial dispersion is observed for each section of the soil to be treated. However, in comparing average removal in each section, no great differences are observed in both tests. Regarding BB2, at the highest electric field the behavior is comparable to that observed for BB1, although with a much lower axial dispersion. However, at the lowest current density a better performance is observed in the soil sections nearer to the compartment in which the surfactant was added, suggesting that transport processes (which are not enhanced in these conditions) are playing a very important role in the removal of diesel from the soil.

Table 1 summarizes the most important parameters to evaluate the efficiency of the electrobioremediation processes combined with biobarriers studied in this work. The most important parameter is the amount of diesel removed from the soil. In this case, in comparing the experiments applying the same electric field, the best results were obtained in the experiments using the BB2 (made with activated sludge microorganisms and the same clay soil). In these experiments high amounts of nutrients were added to the system in the biobarrier area, in order to be sure that nutrients concentrations were not a limiting factor in the technology studied. In this case, due to the addition of the inorganic nutrients in the biobarrier area, where the degradation of the diesel pollutant took part preferentially, lower amounts of them were removed from the system by the electrokinetic processes.

It is possible to compare results obtained applying the technology evaluated in this paper with results previously published studying different strategies for the remediation of the same type of pollution [45]. In this way, in a single biological experiment, for the same duration and very similar experimental conditions than in the experiments presented in this work, an average diesel removal efficiency of $12 \%$ was obtained. On the other hand, in a biological experiment applying a constant value of $1.0 \mathrm{~V} \mathrm{~cm}^{-1}$, conditions obtained in the system, mainly regarding $\mathrm{pH}$ variation, were not adequate for the surveillance of the microbial community. Finally, it was also carried out an electro-bioremediation experiment applying a periodical change (every 24 hours) in the 
polarity of the electric field applied $\left(1.0 \mathrm{~V} \mathrm{~cm}^{-1}\right)$, for which a homogeneous diesel removal efficiency of about $20 \%$ was obtained. Considering these results, we can conclude that by applying the combined biobarrier and electrokinetic treatment suggested in this work an important improvement in the treatment of diesel pollution in soils is obtained.

Finally, it is worth paying attention on the electrical consumption calculated for each experiment. As a general view, lower electrical energy was necessary for the treatment of the soil as compared to the direct electro-bioremediation process $\left(121\right.$ and $1,238 \mathrm{wh} \mathrm{kg}^{-1}$ at 0.5 and $1.0 \mathrm{~V} \mathrm{~cm}^{-1}$, respectively) and the electro-bioremediation process with periodic polarity reversal (42 and 145 wh kg-1 at 0.5 and $1.0 \mathrm{~V} \mathrm{~cm}^{-1}$, respectively). In comparing the removal of diesel and the electrical consumption, the best conditions for the application of the treatment can be established.

From results discussed in this work, it can be concluded that the more efficient treatment consists of the use of a non-specific microbial population that is dispersed in a limited area of the soil treated, in which the best conditions for the biodegradation process were assured, far away from the most extreme conditions derived from the application of the electric field. In this case, a previous microbial acclimation process and biobarrier development was not necessary, making easier the implementation of the treatment.

\section{Conclusions}

The electrokinetic soil flushing combined with biobarriers can be applied successfully for the removal of diesel pollution from clay soil. Optimal conditions for the best performance of the microorganisms degrading the diesel-oil pollutant were achieved by placing the biobarrier in the central section of soil (far to the electrodes). Thus, the negative influence on viability of the culture of the extreme $\mathrm{pH}$ fronts was minimized. The use of a surfactant solution for the transport of the pollutant toward the biodegradation area produces a uniform removal of diesel all over the soil treated. The removal efficiencies obtained were $19.36 \%$ and $27.36 \%$ using the BB1 at 0.5 and $1.0 \mathrm{~V} \mathrm{~cm}^{-1}$, respectively, and $23.33 \%$ and $29.10 \%$ using the $\mathrm{BB} 2$ at 0.5 and $1.0 \mathrm{~V} \mathrm{~cm}^{-1}$, respectively, always after 14 days-long tests. The electrical consumption was also lower using BB2 with energy consumptions below $50{\mathrm{wh} \mathrm{kg}_{\text {soil }}}^{-1}$ during the tests. With all these data, electrobioremediation using the biobarriers approach becomes a very promising technology for the removal of fuels in soil and previous microbial acclimation process for biobarrier development is not necessary but just direct application of activated sludge obtained in a municipal WWTP, making easier the implementation of the treatment technology.

\section{Acknowledgements}


The authors acknowledge funding support from the EU and Spanish Government through the MINECO Project CTM2013-45612-R and INNOCAMPUS. 


\section{Literature cited}

[1] A.T. Yeung, Y.-Y. Gu, A review on techniques to enhance electrochemical remediation of contaminated soils, J. Hazard. Mater., 195 (2011) 11.

[2] J.J. Ortega-Calvo, M.C. Tejeda-Agredano, C. Jimenez-Sanchez, E. Congiu, R. Sungthong, J.L. NiquiArroyo, M. Cantos, Is it possible to increase bioavailability but not environmental risk of PAHs in bioremediation?, J. Hazard. Mater., 261 (2013) 733.

[3] B. Erable, N.M. Duţeanua, M.M. Ghangrekar, C. Dumas, K. Scott, Application of electro-active biofilms, Biofouling, 26 (2010) 57.

[4] M. Romantschuk, I. Sarand, T. Petänen, R. Peltola, M. Jonsson-Vihanne, T. Koivula, K. Yrjälä, K. Haahtela, Means to improve the effect of in situ bioremediation of contaminated soil: An overview of novel approaches, Environ. Pollut., 107 (2000) 179.

[5] M. Megharaj, B. Ramakrishnan, K. Venkateswarlu, N. Sethunathan, R. Naidu, Bioremediation approaches for organic pollutants: A critical perspective, Environ. Int., 37 (2011) 1362.

[6] M. Pazos, E. Rosales, T. Alcántara, J. Gómez, M.A. Sanromán, Decontamination of soils containing PAHs by electroremediation: A review, J. Hazard. Mater., 177 (2010) 1.

[7] R.T. Gill, M.J. Harbottle, J.W.N. Smith, S.F. Thornton, Electrokinetic-enhanced bioremediation of organic contaminants: A review of processes and environmental applications, Chemosphere, 107 (2014) 31.

[8] D. Huang, Q. Xu, J. Cheng, X. Lu, H. Zhang, Electrokinetic remediation and its combined technologies for removal of organic pollutants from contaminated soils, Int. J. Electrochem. Sc., 7 (2012) 4528.

[9] H.I. Gomes, C. Dias-Ferreira, A.B. Ribeiro, Electrokinetic remediation of organochlorines in soil: Enhancement techniques and integration with other remediation technologies, Chemosphere, 87 (2012) 1077.

[10] L.Y. Wick, L. Shi, H. Harms, Electro-bioremediation of hydrophobic organic soil-contaminants: A review of fundamental interactions, Electrochim. Acta, 52 (2007) 3441.

[11] G.V. Chilingar, W.W. Loo, L.F. Khilyuk, S.A. Katz, Electrobioremediation of soils contaminated with hydrocarbons and metals: Progress report, Energ. Source., 19 (1997) 129.

[12] K.R. Reddy, C. Cameselle, Electrochemical Remediation Technologies for Polluted Soils, Sediments and Groundwater, John Wiley \& Sons, Inc., Hoboken, New Jersey.2009.

[13] A. Careghini, S. Saponaro, E. Sezenna, Biobarriers for groundwater treatment: A review, Water Sci. Technol., 67 (2013) 453.

[14] M.M. Scherer, S. Richter, R.L. Valentine, P.J.J. Alvarez, Chemistry and microbiology of permeable reactive barriers for in situ groundwater clean up, CRC Cr. Rev. Microbiol., 26 (2000) 221.

[15] E.K. Nyer, In situ treatment technology, Lewis Publishers, Boca Raton (Fla.), London, New York, 2001.

[16] R. Focht, J. Vogan, S. O'Hannesin, Field application of reactive iron walls for in-situ degradation of volatile organic compounds in groundwater, Remed. J., 6 (1996) 81.

[17] A.R. Gavaskar, Design and construction techniques for permeable reactive barriers, J. Hazard. Mater., 68 (1999) 41.

[18] B.D.M. Painter, Reactive barriers: Hydraulic performance and design enhancements, Ground Water, 42 (2004) 609.

[19] F.G. Simon, T. Meggyes, T. Tünnerrneier, K. Czurda, K.E. Roehl, Long-term behaviour of permeable reactive barriers used for the remediation of contaminated groundwater, in: 8th International Conference on Radiactive Waste Management and Environmental Remediation, ICEM, 2001, Bruges, Belgium, 30 September 2001 through 4 October 2001, Vol. 1, p. 637.

[20] R. Thiruvenkatachari, S. Vigneswaran, R. Naidu, Permeable reactive barrier for groundwater remediation, J. Ind. Eng. Chem., 14 (2008) 145.

[21] H.I. Chung, M. Lee, A new method for remedial treatment of contaminated clayey soils by electrokinetics coupled with permeable reactive barriers, Electrochim. Acta, 52 (2007) 3427.

[22] L. Cang, D.M. Zhou, D.Y. Wu, A.N. Alshawabkeh, Coupling electrokinetics with permeable reactive barriers of zero-valent iron for treating a chromium contaminated soil, Separ. Sci. Technol., 44 (2009) 2188.

[23] Y.C. Huang, Y.W. Cheng, Electrokinetic-enhanced nanoscale iron reactive barrier of trichloroethylene solubilized by Triton X-100 from groundwater, Electrochim. Acta, 86 (2012) 177.

[24] Z. Li, S. Yuan, J. Wan, H. Long, M. Tong, A combination of electrokinetics and Pd/Fe PRB for the remediation of pentachlorophenol-contaminated soil, J. Contam. Hydrol., 124 (2011) 99.

[25] C. Ruiz, E. Mena, P. Cañizares, J. Villaseñor, M.A. Rodrigo, Removal of 2,4,6-trichlorophenol from spiked clay soils by electrokinetic soil flushing assisted with granular activated carbon permeable reactive barrier, Ind. Eng. Chem., 53 (2014) 840.

[26] J. Wan, Z. Li, X. Lu, S. Yuan, Remediation of a hexachlorobenzene-contaminated soil by surfactantenhanced electrokinetics coupled with microscale Pd/Fe PRB, J. Hazard. Mater., 184 (2010) 184.

[27] C. Yuan, T.S. Chiang, The mechanisms of arsenic removal from soil by electrokinetic process coupled with iron permeable reaction barrier, Chemosphere, 67 (2007) 1533.

[28] C.M. Kao, S.C. Chen, J.K. Liu, Development of a biobarrier for the remediation of PCE-contaminated aquifer, Chemosphere, 43 (2001) 1071.

[29] C.M. Kao, L. Yang, Enhanced bioremediation of trichloroethene contaminated by a biobarrier system, Water Sci. Technol.,42 (2000) 429. 
[30] S.J. Liu, B. Jiang, G.Q. Huang, X.G. Li, Laboratory column study for remediation of MTBE-contaminated groundwater using a biological two-layer permeable barrier, Water Res., 40 (2006) 3401.

[31] H.Q. Ma, L.Y. Zhang, H.L. Zhang, S. Li, In situ remediation of petroleum hydrocarbon contaminated groundwater using a novel biobarrier, Chongqing Daxue Xuebao/Journal of Chongqing University, 34 (2011) 99.

[32] K.D. Miller, P.C. Johnson, C.L. Bruce, Full-scale in-situ biobarrier demonstration for containment and treatment of MTBE, Remed. J., 12 (2001) 25.

[33] S. Saponaro, M. Negri, E. Sezenna, L. Bonomo, C. Sorlini, Groundwater remediation by an in situ biobarrier: A bench scale feasibility test for methyl tert-butyl ether and other gasoline compounds, J. Hazard. Mater., 167 (2009) 545-552.

[34] S. Saponaro, A. Careghini, L. Romele, E. Sezenna, A. Franzetti, I. Gandolfi, M. Daghio, G. Bestetti, Remediation of groundwater polluted by gasoline-derived compounds with biobarriers, WIT Trans. Ecol. Envir., 164 (2012) 439.

[35] E.M. Ramírez, C.S. Jiménez, J.V. Camacho, M.A. Rodrigo, P. Cañizares, Feasibility of coupling permeable bio-barriers and electrokinetics for the treatment of diesel hydrocarbons polluted soils, Electrochim. Acta, (2015) 192.

[36] E. Mena, C. Ruiz, J. Villaseñor, M.A. Rodrigo, P. Cañizares, Biological permeable reactive barriers coupled with electrokinetic soil flushing for the treatment of diesel-polluted clay soil, J. Hazard. Mater., 283 (2015) 131.

[37] S.T. Lohner, D. Katzoreck, A. Tiehm, Electromigration of microbial electron acceptors and nutrients: (I) Transport in synthetic media, J. Environ. Sci. Heal. A, 43 (2008) 913.

[38] S.T. Lohner, D. Katzoreck, A. Tiehm, Electromigration of microbial electron acceptors and nutrients: (II) Transport in groundwater, J. Environ. Sci. Heal. A, 43 (2008) 922.

[39] K. Maturi, K.R. Reddy, C. Cameselle, Surfactant-enhanced electrokinetic remediation of mixed contamination in low permeability soil, Separ. Sci. Technol., 44 (2009) 2385.

[40] E. Moliterni, R.G. Jiménez-Tusset, M. Villar Rayo, L. Rodriguez, F.J. Fernández, J. Villaseñor, Int. J. Environ. Sci. Te., 9 (2012) 749.

[41] E. Mena Ramírez, J. Villaseñor Camacho, M.A. Rodrigo Rodrigo, P. Cañizares Cañizares, Feasibility of electrokinetic oxygen supply for soil bioremediation purposes, Chemosphere, 117 (2014) 382.

[42] R. López-Vizcaíno, J. Alonso, P. Cañizares, M.J. León, V. Navarro, M.A. Rodrigo, C. Sáez, Electroremediation of a natural soil polluted with phenanthrene in a pilot plant, J. Hazard. Mater., 265 (2014) 142.

[43] R. López-Vizcaíno, J. Alonso, P. Cañizares, M.J. León, V. Navarro, M.A. Rodrigo, C. Sáez, Removal of phenanthrene from synthetic kaolin soils by eletrokinetic soil flushing, Sep. Purif. Technol., 132 (2014) 33.

[44] E. Moliterni, L. Rodriguez, F.J. Fernández, J. Villaseñor, Feasibility of different bioremediation strategies for treatment of clayey and silty soils recently polluted with diesel hydrocarbons, Water Air Soil Poll., 223 (2012) 2473.

[45] E.M. Ramírez, J.V. Camacho, M.A. Rodrigo, P. Cañizares, Combination of bioremediation and electrokinetics for the in-situ treatment of diesel polluted soil: A comparison of strategies, Sci. Total Environ., 533 (2015) 307. 


\section{Figure captions}

Figure 1: Bench scale setup scheme.

Figure 2: Time-course of the current density in the electro-bioremediation tests with biobarriers. Experiments with BB1 at $0.5 \mathrm{~V} \mathrm{~cm}^{-1}(\square)$ and $1.0 \mathrm{~V} \mathrm{~cm}^{-1}(\mathbf{\square})$ and experiments with BB2 at $0.5 \mathrm{~V}$ $\mathrm{cm}^{-1}(\bigcirc)$ and $1.0 \mathrm{~V} \mathrm{~cm}^{-1}(\mathbf{O})$.

Figure 3: Time-course of the electro-osmotic flow during the electro-bioremediation tests with biobarriers. Experiments with BB1 at $0.5 \mathrm{~V} \mathrm{~cm}^{-1}(\square)$ and $1.0 \mathrm{~V} \mathrm{~cm}^{-1}(\mathbf{\square})$ and experiments with $\mathrm{BB} 2$ at $0.5 \mathrm{~V} \mathrm{~cm}^{-1}(\bigcirc)$ and $1.0 \mathrm{~V} \mathrm{~cm}^{-1}(\bigcirc)$.

Figure 4: Time-course of the temperature (a), $\mathrm{pH}(\mathrm{b})$, dissolved oxygen concentration (c) and conductivity (d) in the biobarrier zone in the electro-bioremediation tests with biobarriers. Experiments with BB1 at $0.5 \mathrm{~V} \mathrm{~cm}^{-1}(\square)$ and $1.0 \mathrm{~V} \mathrm{~cm}^{-1}(\mathbf{\square})$ and experiments with BB2 at $0.5 \mathrm{~V}$ $\mathrm{cm}^{-1}(\bigcirc)$ and $1.0 \mathrm{~V} \mathrm{~cm}^{-1}(\bigcirc)$.

Figure 5: Time-course of the $\mathrm{pH}$ of the anolyte (a) and catholyte (b) during the electrobioremediation tests with biobarriers. Experiments with BB1 at $0.5 \mathrm{~V} \mathrm{~cm}^{-1}(\square)$ and $1.0 \mathrm{~V} \mathrm{~cm}^{-1}$ (ם) and experiments with BB2 at $0.5 \mathrm{~V} \mathrm{~cm}^{-1}(\bigcirc)$ and $1.0 \mathrm{~V} \mathrm{~cm}^{-1}(\bullet)$.

Figure 6: Time-course of the conductivity of the anolyte (a) and catholyte (b) during the direct electro-bioremediation tests with biobarriers. Experiments with BB1 at $0.5 \mathrm{~V} \mathrm{~cm}^{-1}(\square)$ and $1.0 \mathrm{~V}$ $\mathrm{cm}^{-1}(\mathbf{\square})$ and experiments with $\mathrm{BB} 2$ at $0.5 \mathrm{~V} \mathrm{~cm}^{-1}(\bigcirc)$ and $1.0 \mathrm{~V} \mathrm{~cm}^{-1}(\mathbf{O})$.

Figure 7: Moisture profile in the soil at the start (- -$)$ and at the end ( - ) of the electrobioremediation tests with biobarriers. Experiments with $\mathrm{BB} 1$ at $0.5 \mathrm{~V} \mathrm{~cm}^{-1}$ (a) and $1.0 \mathrm{~V} \mathrm{~cm}^{-1}$ (b) and experiments with BB2 at $0.5 \mathrm{~V} \mathrm{~cm}^{-1}$ (c) and $1.0 \mathrm{~V} \mathrm{~cm}^{-1}(\mathrm{~d})$. The line (-) is the average of the four values in the different axial positions (top right $(\bullet)$, top left $(\boldsymbol{\square})$, bottom right $(\boldsymbol{\Delta})$, bottom left $(\bullet))$.

Figure 8: Conductivity profile in the soil at the start (- $)$ and at the end ( - ) of the electrobioremediation tests with biobarriers. Experiments with $\mathrm{BB} 1$ at $0.5 \mathrm{~V} \mathrm{~cm}^{-1}$ (a) and $1.0 \mathrm{~V} \mathrm{~cm}^{-1}$ (b) and experiments with $\mathrm{BB} 2$ at $0.5 \mathrm{~V} \mathrm{~cm}^{-1}(\mathrm{c})$ and $1.0 \mathrm{~V} \mathrm{~cm}^{-1}(\mathrm{~d})$. The line (-) is the average of the four values in the different axial positions (top right $(\bullet)$, top left $(\boldsymbol{\square})$, bottom right $(\boldsymbol{\Delta})$, bottom left $(\mathbf{O})$ ).

Figure 9: $\mathrm{pH}$ profile in the soil at the start $(--)$ and at the end (-) of the electrobioremediation tests with biobarriers. Experiments with $\mathrm{BB} 1$ at $0.5 \mathrm{~V} \mathrm{~cm}^{-1}$ (a) and $1.0 \mathrm{~V} \mathrm{~cm}^{-1}$ (b) and experiments with BB2 at $0.5 \mathrm{~V} \mathrm{~cm}^{-1}$ (c) and $1.0 \mathrm{~V} \mathrm{~cm}^{-1}(\mathrm{~d})$. The line (-) is the average of the four values in the different axial positions (top right $(\bullet)$, top left $(\boldsymbol{\square})$, bottom right $(\boldsymbol{\Delta})$, bottom left $(\bullet)$ ).

Figure 10: Microorganisms concentration profile in the soil at the end ( - ) of the electrobioremediation tests with biobarriers. Experiments with $\mathrm{BB} 1$ at $0.5 \mathrm{~V} \mathrm{~cm}^{-1}$ (a) and $1.0 \mathrm{~V} \mathrm{~cm}^{-1}$ (b) and experiments with BB2 at $0.5 \mathrm{~V} \mathrm{~cm}^{-1}(\mathrm{c})$ and $1.0 \mathrm{~V} \mathrm{~cm}^{-1}(\mathrm{~d})$. The line (-) is the average of 
the four values in the different axial positions (top right $(\bullet)$, top left $(\boldsymbol{\square})$, bottom right $(\boldsymbol{\Delta})$, bottom left (○)).

Figure 11: Surfactant concentration profile in the soil at the start (- ) and at the end ( - ) of the electro-bioremediation tests with biobarriers. Experiments with $\mathrm{BB} 1$ at $0.5 \mathrm{~V} \mathrm{~cm}^{-1}$ (a) and 1.0 $\mathrm{V} \mathrm{cm}^{-1}$ (b) and experiments with $\mathrm{BB} 2$ at $0.5 \mathrm{~V} \mathrm{~cm}^{-1}$ (c) and $1.0 \mathrm{~V} \mathrm{~cm}^{-1}(\mathrm{~d})$. The line (-) is the average of the four values in the different axial positions (top right ( $\bullet$ ), top left $(\mathbf{\square})$, bottom right

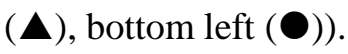

Figure 12: Diesel concentration profile in the soil at the start (- ) and at the end (- $\longrightarrow$ of the electro-bioremediation tests with biobarriers. Experiments with $\mathrm{BB} 1$ at $0.5 \mathrm{~V} \mathrm{~cm}^{-1}$ (a) and $1.0 \mathrm{~V}$ $\mathrm{cm}^{-1}$ (b) and experiments with $\mathrm{BB} 2$ at $0.5 \mathrm{~V} \mathrm{~cm}^{-1}$ (c) and $1.0 \mathrm{~V} \mathrm{~cm}^{-1}(\mathrm{~d})$. The line (-) is the average of the four values in the different axial positions (top right $(\bullet)$, top left $(\mathbf{\square})$, bottom right $(\mathbf{\Delta})$, bottom left $(\mathbf{O})$ ). 


\section{$\underline{\text { Figures }}$}

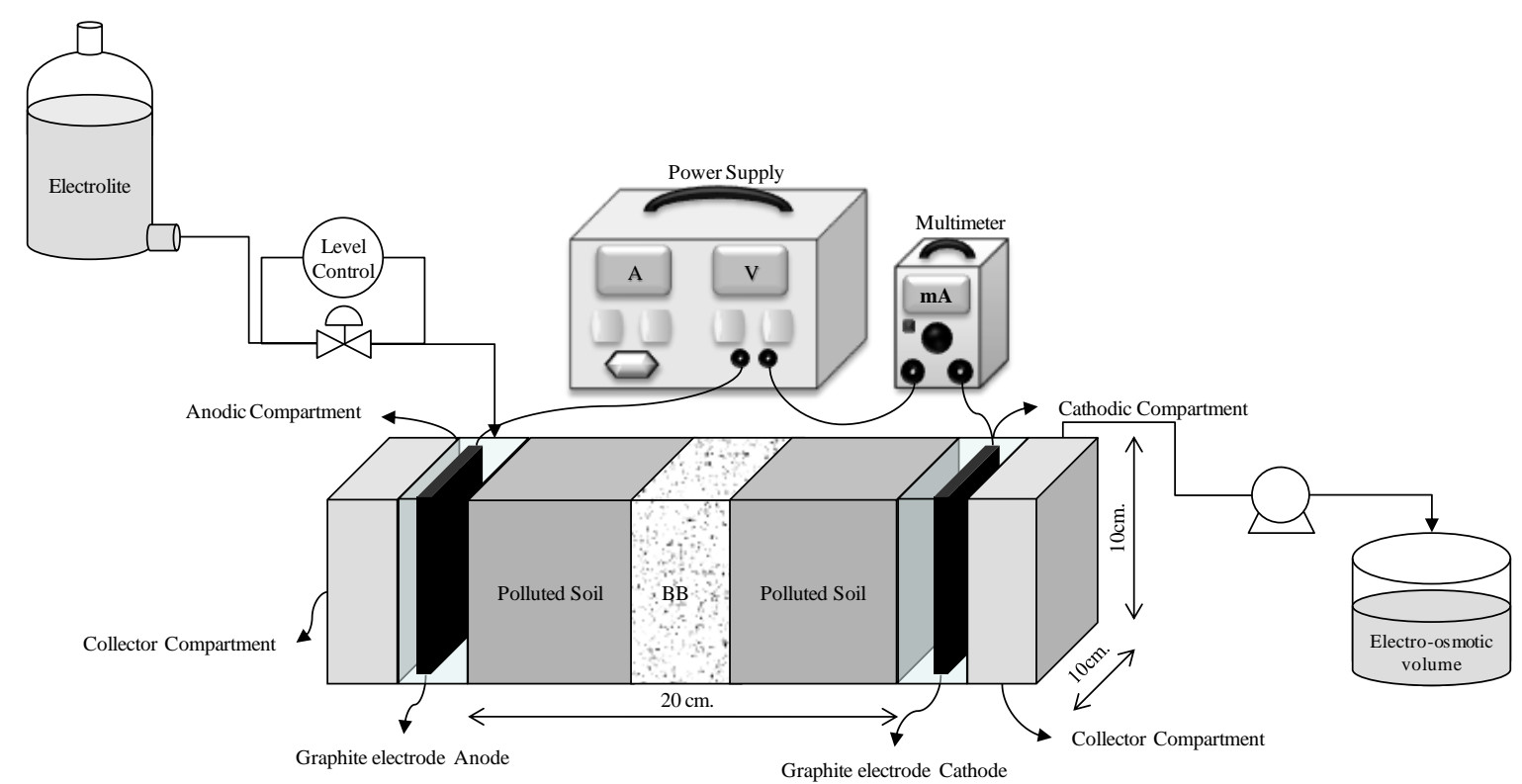

Figure 1. 


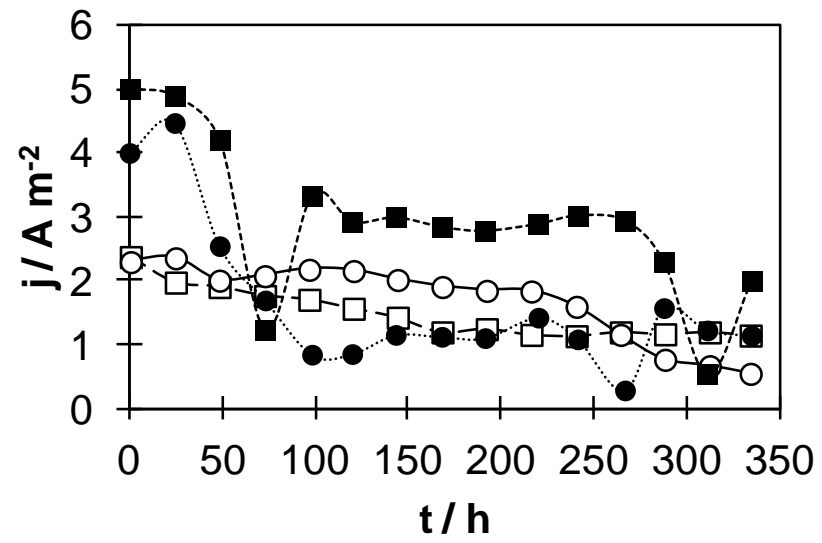

Figure 2. 


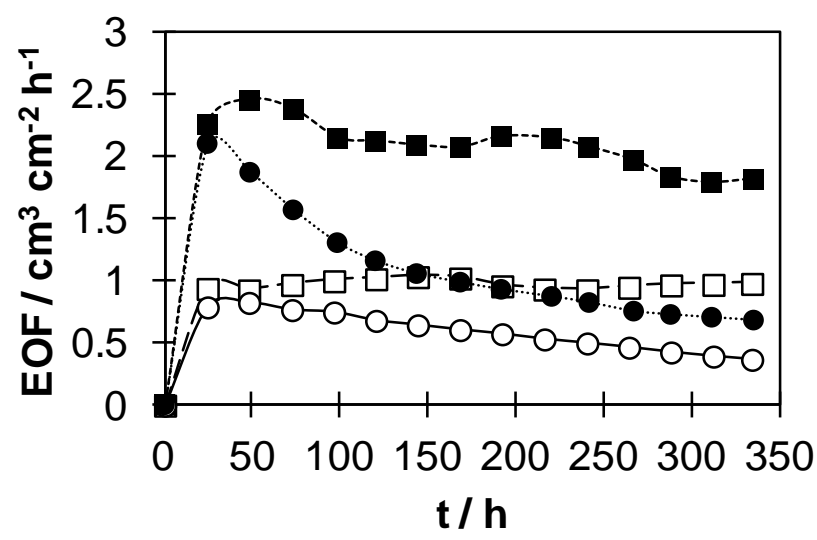

Figure 3. 

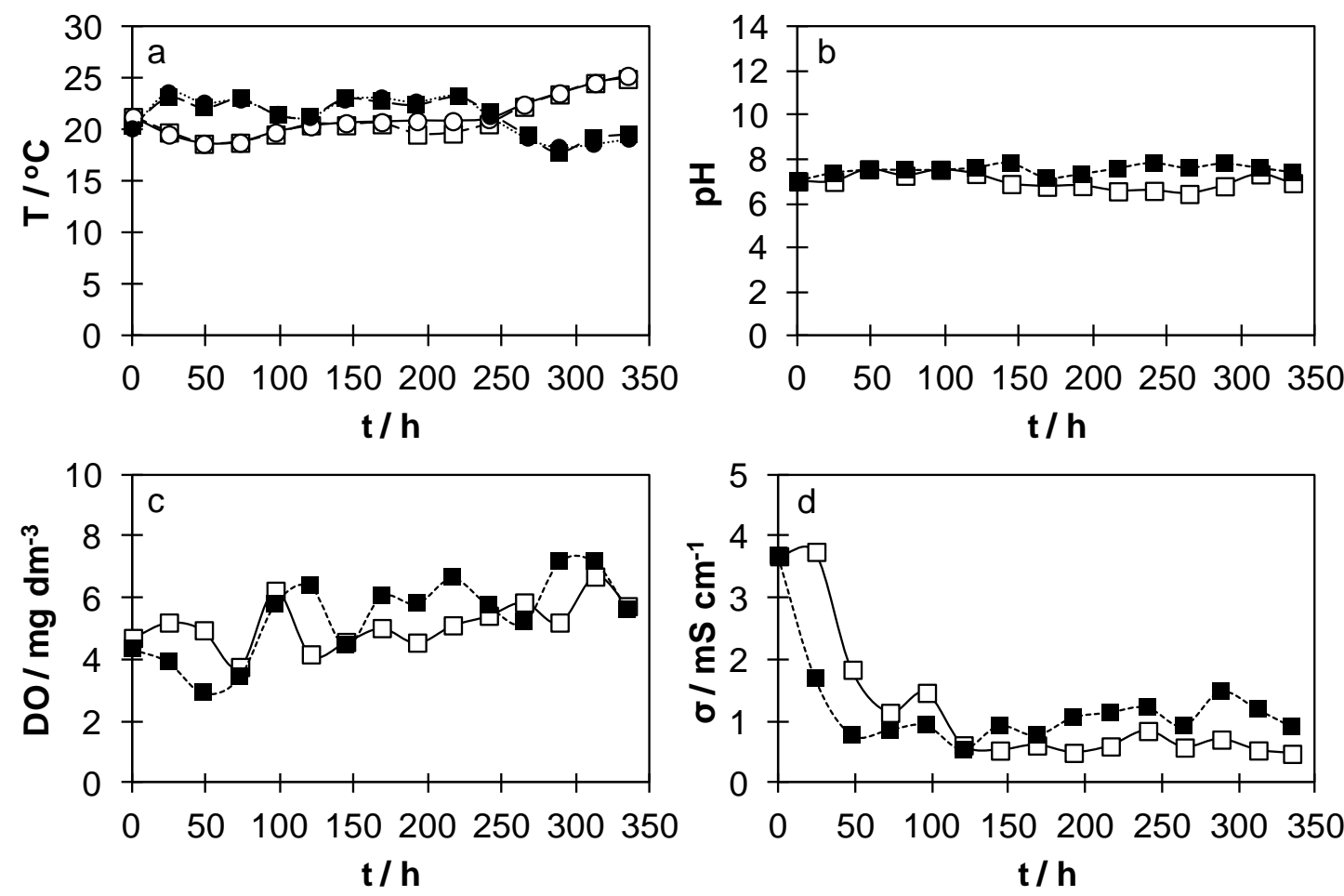

Figure 4. 

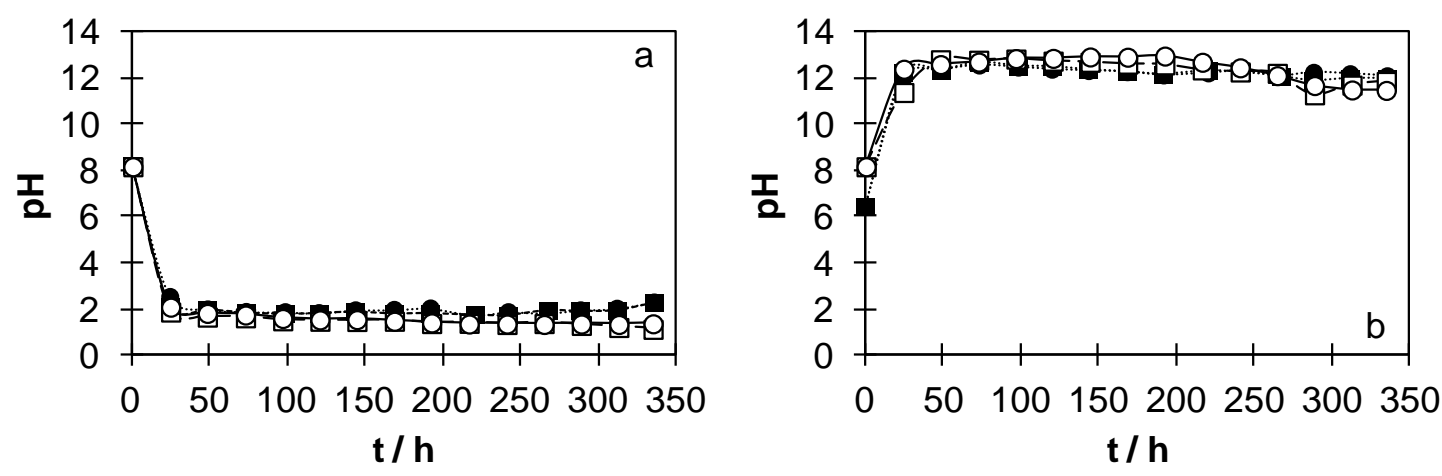

Figure 5. 

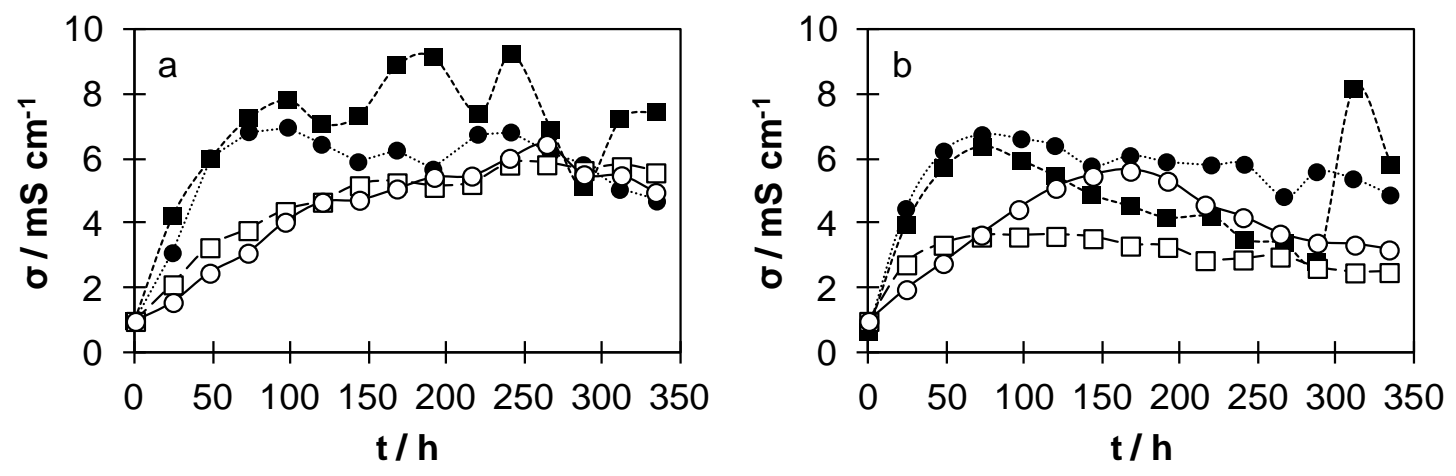

Figure 6. 

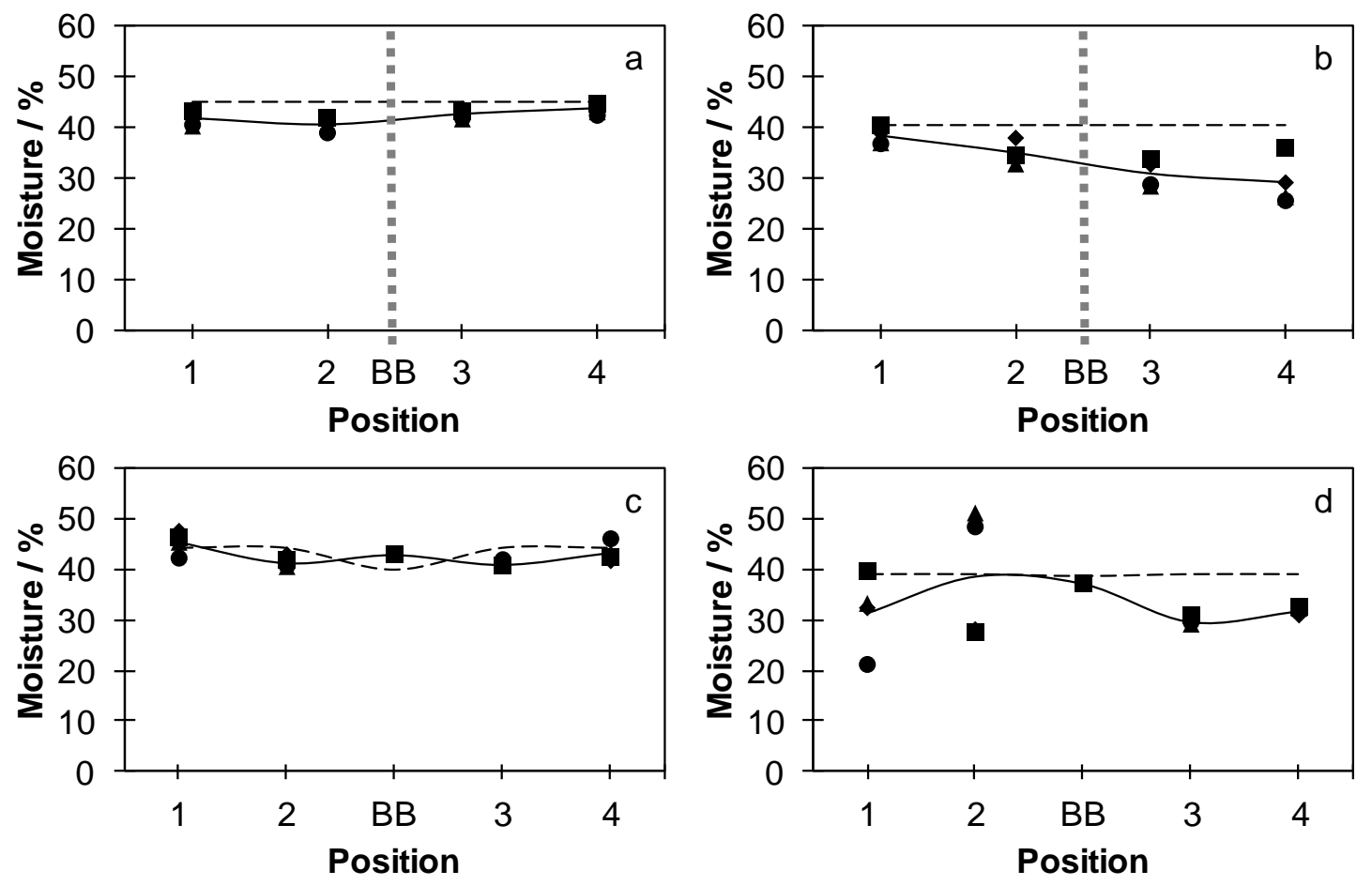

Figure 7. 

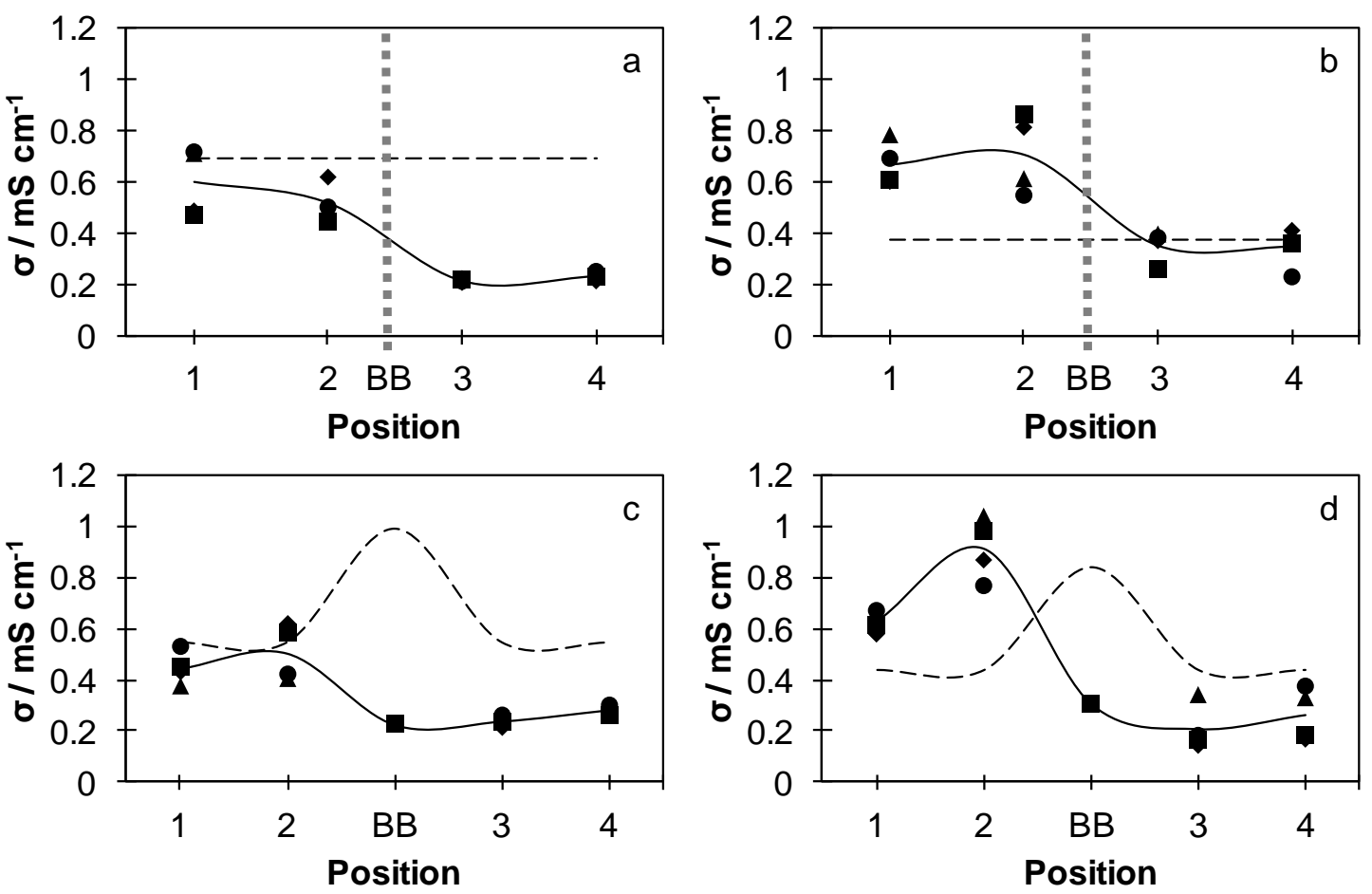

Figure 8. 

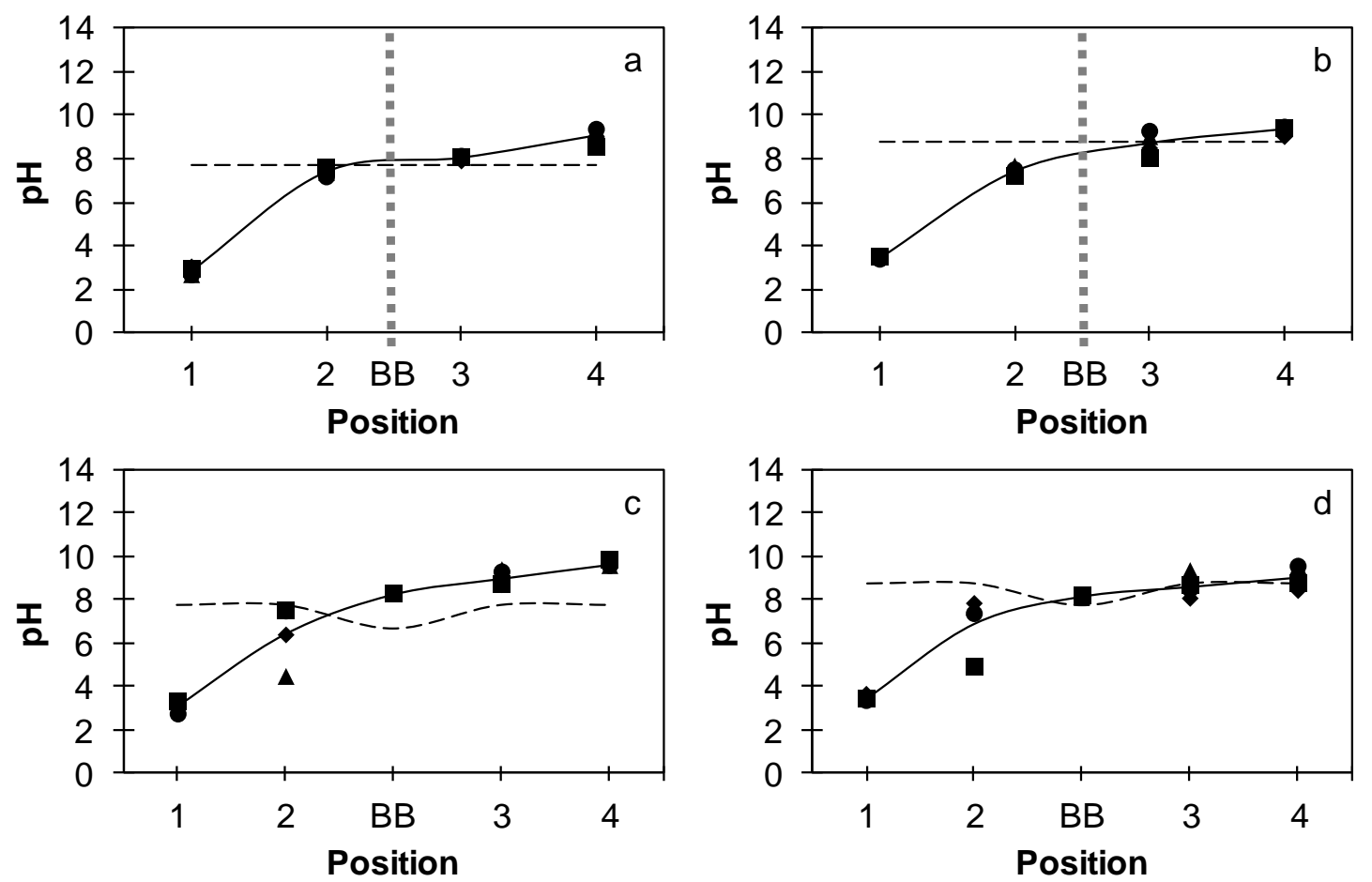

Figure 9. 

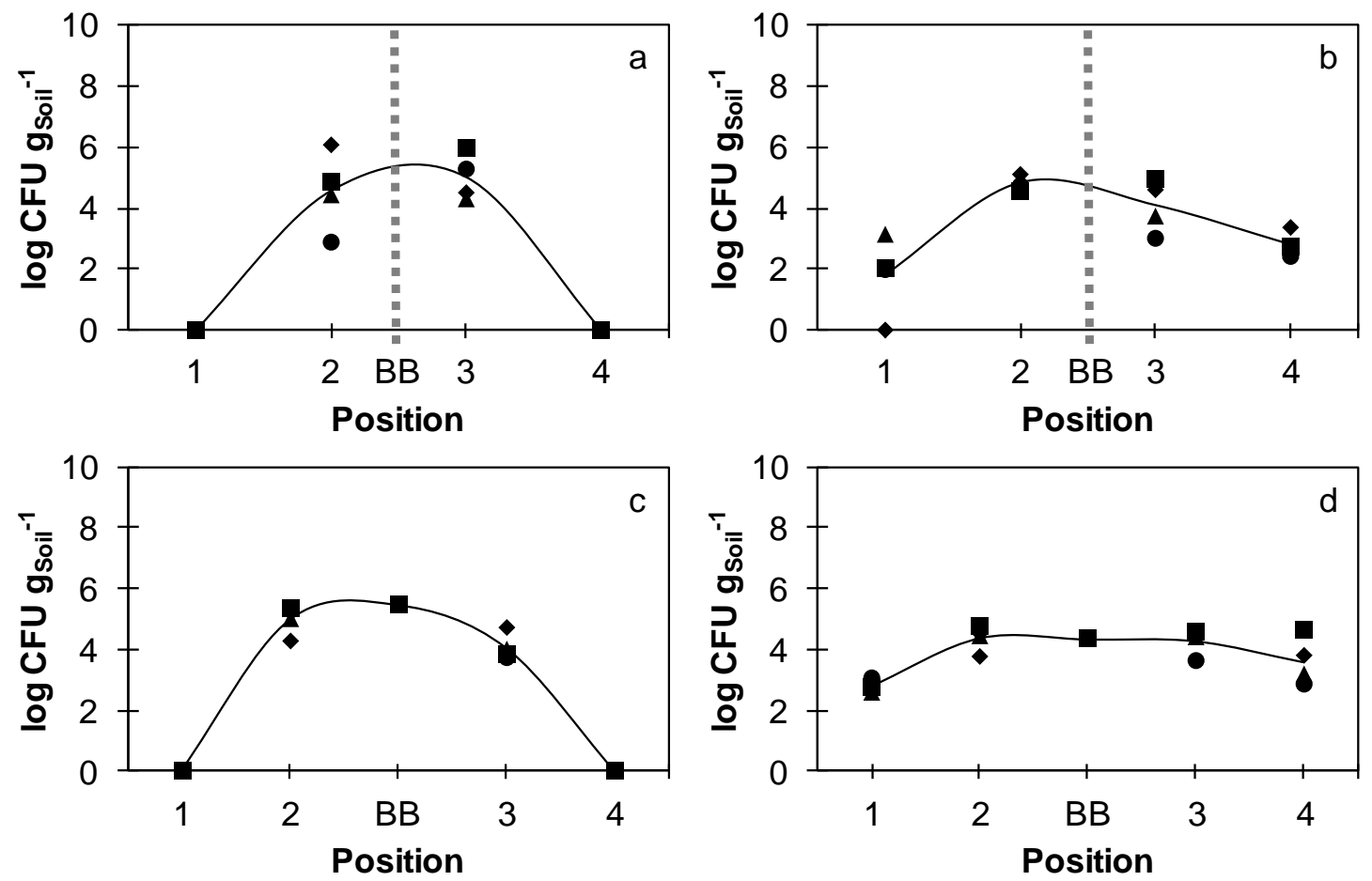

Figure 10. 

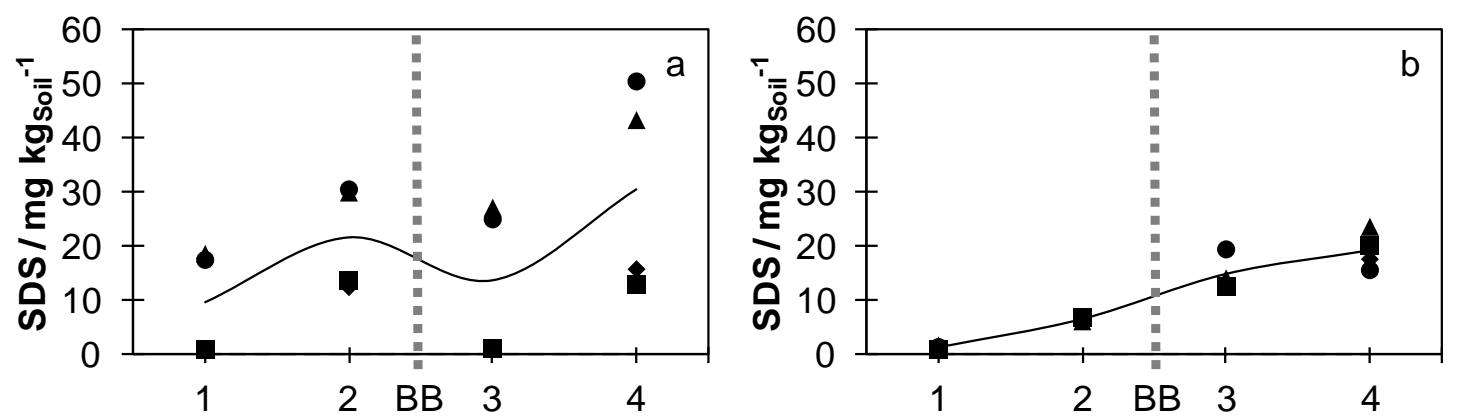

Position
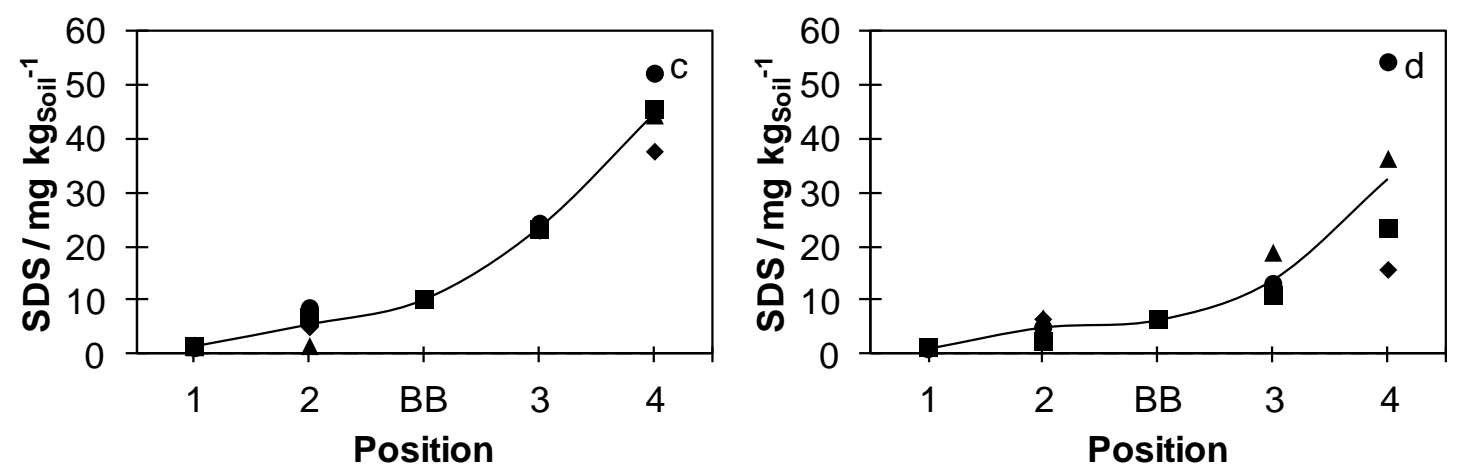

Figure 11. 

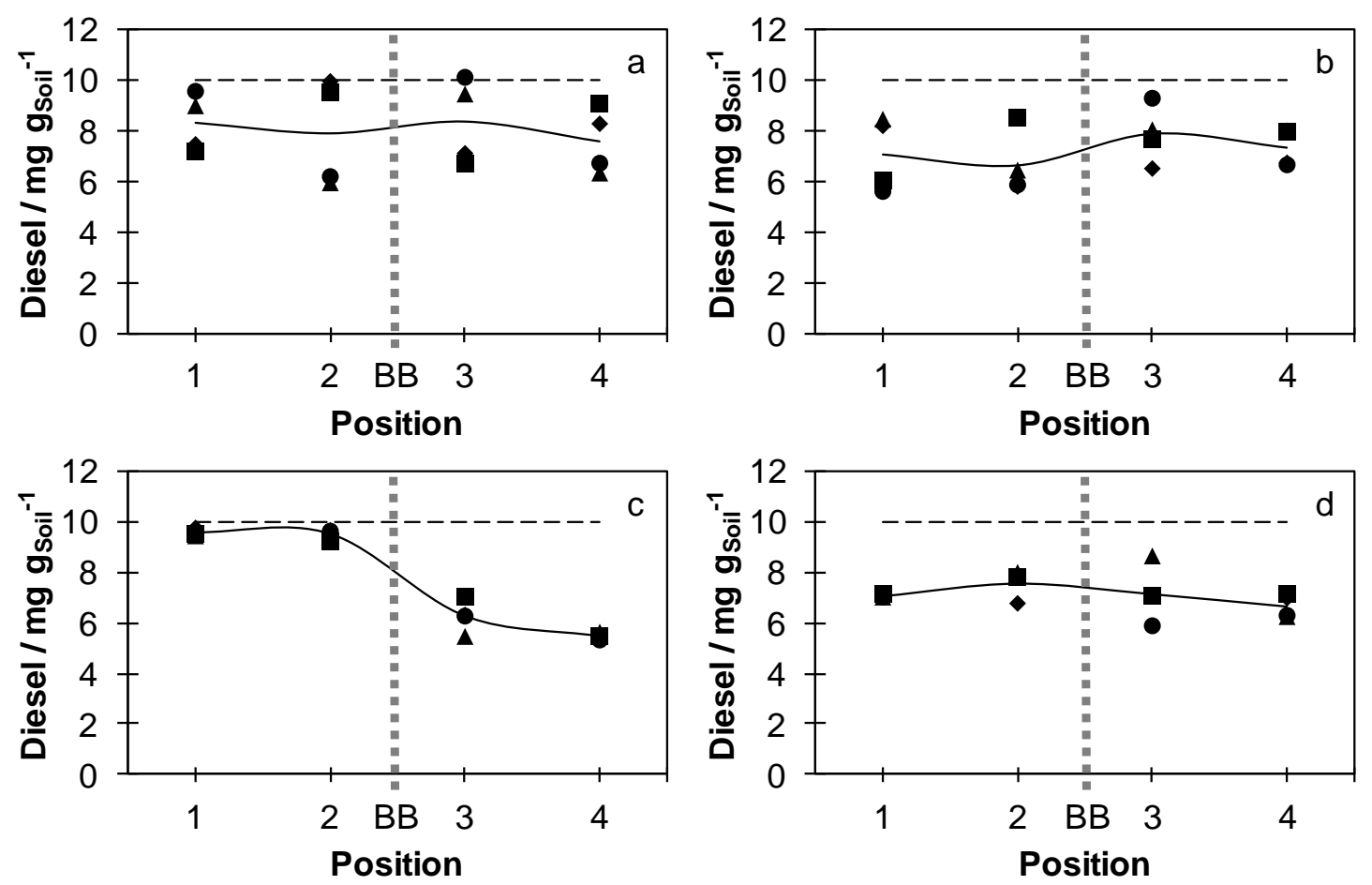

Figure 12. 
Table 1: Pollutant, inorganic nutrients and electrical consumption in the electro-bioremediation tests with biobarriers.

\begin{tabular}{|c|c|c|c|c|}
\hline & \multicolumn{4}{|c|}{ Test } \\
\hline & $\begin{array}{c}\mathrm{BB1} \\
0.5 \mathrm{~V} \mathrm{~cm}^{-1}\end{array}$ & $\begin{array}{c}\mathrm{BB1} \\
1.0 \mathrm{~V} \mathrm{~cm}^{-1}\end{array}$ & $\begin{array}{c}\mathrm{BB2} \\
0.5 \mathrm{~V} \mathrm{~cm}^{-1}\end{array}$ & $\begin{array}{c}\mathrm{BB2} \\
1.0 \mathrm{~V} \mathrm{~cm}^{-1}\end{array}$ \\
\hline $\begin{array}{c}\text { Soil treated } \\
\mathrm{g}\end{array}$ & 2,505 & 2,567 & 2,406 & 2,564 \\
\hline $\begin{array}{l}\text { Diesel added } \\
\mathrm{mg}^{\mathrm{mg} \mathrm{kg}^{-1} \text { Soil }}\end{array}$ & $\begin{array}{l}25,049 \\
10,000\end{array}$ & $\begin{array}{l}25,668 \\
10,000\end{array}$ & $\begin{array}{l}24,058 \\
10,000\end{array}$ & $\begin{array}{l}25,642 \\
10,000\end{array}$ \\
\hline $\begin{array}{l}\text { Moisture } \\
\mathrm{mL} \mathrm{kg}_{\text {Soil }^{-1}}\end{array}$ & 450 & 406 & 442 & 406 \\
\hline $\begin{array}{l}\mathbf{N}_{\text {Total }} \text { added } \\
\mathrm{mg}^{\mathrm{mg} \mathrm{kg}_{\text {Soil }}^{-1}}\end{array}$ & $\begin{array}{l}705 \\
282\end{array}$ & $\begin{array}{l}981 \\
382\end{array}$ & $\begin{array}{c}1,404 \\
584\end{array}$ & $\begin{array}{l}802 \\
313\end{array}$ \\
\hline $\begin{array}{l}\mathbf{P}_{\text {Total }} \text { added } \\
\mathrm{mg} \\
\mathrm{mg} \mathrm{kg}_{\text {Soil }^{-1}}\end{array}$ & $\begin{array}{c}197 \\
79\end{array}$ & $\begin{array}{l}293 \\
114\end{array}$ & $\begin{array}{c}184 \\
76\end{array}$ & $\begin{array}{c}120 \\
47\end{array}$ \\
\hline $\begin{array}{l}\text { Flushing fluid added } \\
\qquad \mathrm{mL} \\
\mathrm{mL} \mathrm{kg}_{\text {Soil }^{-1}}\end{array}$ & $\begin{array}{c}3,563 \\
703\end{array}$ & $\begin{array}{l}5,222 \\
2,034\end{array}$ & $\begin{array}{c}2,139 \\
889\end{array}$ & $\begin{array}{l}2,700 \\
1,053\end{array}$ \\
\hline $\begin{array}{c}\text { Diesel removed } \\
\mathrm{mg} \\
\%\end{array}$ & $\begin{array}{l}4,850 \\
19.36\end{array}$ & $\begin{array}{l}7,023 \\
27.36\end{array}$ & $\begin{array}{l}5,613 \\
23.33\end{array}$ & $\begin{array}{l}7,461 \\
29.10\end{array}$ \\
\hline $\begin{array}{c}\mathbf{N}_{\text {Total }} \text { removed } \\
\text { Electrodic compartments } \\
\text { and sampling } \\
\mathrm{mg}\end{array}$ & 45 & 20 & 16 & 22 \\
\hline $\begin{array}{c}\mathbf{P}_{\text {Total }} \text { removed } \\
\text { Electrodic compartments } \\
\text { and sampling } \\
\mathrm{mg}\end{array}$ & 1 & 3 & 1 & 3 \\
\hline $\begin{array}{c}\text { Flushing fluid removed } \\
\text { Electro-osmosis } \\
\text { Sampling } \\
\mathrm{mL}\end{array}$ & $\begin{array}{c}1,236 \\
420\end{array}$ & $\begin{array}{c}2,412 \\
372\end{array}$ & $\begin{array}{l}414 \\
280\end{array}$ & $\begin{array}{l}825 \\
280\end{array}$ \\
\hline $\begin{array}{l}\text { Electrical consumption } \\
\qquad \mathrm{Wh} \mathrm{kg}_{\text {Soil }^{-1}}\end{array}$ & 21 & 90 & 23 & 48 \\
\hline
\end{tabular}

